\title{
ON INITIAL BOUNDARY VALUE PROBLEMS FOR THE DEGENERATE 1D WAVE EQUATION
}

\author{
Vladimir L. Borsch* \\ Communicated by Prof. P. I. Kogut
}

\begin{abstract}
Initial boundary value problems in space-time rectangle $[0, T] \times[-l,+l]$ for the following linear inhomogeneous degenerate wave equation of the second order

$$
\frac{\partial^{2} u}{\partial t^{2}}-\frac{\partial}{\partial x}\left(a \frac{\partial u}{\partial x}\right)=g
$$

are considered. The degeneracy of the equation means that continuous and piecewise smooth coefficient function $a(x)$ vanishes in single points of segment $[-l,+l]$.

The well-posedness of the initial boundary value problems is achieved using some approaches to regularization of the equation and the theory of characteristics. The problems for wave equation are then reduced to problems for hyperbolic balance laws $2 \times 2$ and $3 \times 3$ of partial differential equations of the first order. Weak solutions to the problems are obtained using proper numerical methods.

Results obtained for some approaches to regularization are presented.
\end{abstract}

Key words: degenerate wave equations, the theory of characteristics.

2010 Mathematics Subject Classification: 35L05, 35L35, 35L80.

\section{Introduction}

"The earth was without form, and void..." Gen 1:2

In July of 2019 Prof. P. I. Kogut involved the author of the current study into a discussion concerning a degenerate spatially one-dimensional wave equation of the second order and admissible well-posed initial boundary value problems for such an equation. Shortly, the degeneracy of the wave equation means vanishing of the coefficient $a(x)$ of the highest order spatial derivative in some intervals of spatial variable $x$, or 'lacunae' $\{x: a(x)=0\}$, and changing the type of the wave equation in the lacunas from hyperbolic to parabolic.

Solutions to admissible well-posed initial boundary value problems for the wave equation with no degeneracy are known to be composed of two waves running in the opposite directions, and the local squared velocities of the waves equal to the local values of the coefficient $a(x)$. Vanishing of the coefficient $a(x)$ no doubt prevent waves from running inside lacunae. But do the waves running outside

\footnotetext{
*Dept. of Differential Equations, Faculty of Mech \& Math, Oles Honchar Dnipro National University, 72, Gagarin av., Dnipro, 49010, Ukraine, bvı@dsu.dp.ua

(C) Vladimir L. Borsch, 2019.
} 
the lacunae reflect from the lacunae or are the waves damped by the lacunae? How to pose properly initial boundary value problems in case of lacunae?

Thanks courtesy of Prof. P. I. Kogut, draft copy of his article [4] had been a part of the discussion. The articles deals with the existence, non-uniqueness and a priori estimates of the solutions to well-posed initial boundary value problems for the degenerate wave equation. Unfortunately, none of the solutions was given in an explicit form. Hence, it was attempted to find solutions using exceptionally classical direct approaches. The current study stems from those attempts. By classical approaches we imply here: 1) the theory of characteristics for linear partial differential equations in two independent variables of the second order, 2) the theory of weak solutions to initial boundary value problems for the former equations and for hyperbolic systems of linear partial differential equations of the first order, and 3) numerical methods for obtaining such weak solutions.

The article is arranged as follows.

In section 2 we define exactly what we imply by the degenerate wave equation and then discuss preliminaries of initial boundary value problems for the equation.

In section 3 we implement characteristic analysis of the degenerate wave equation to well-pose initial boundary value problems for the equation.

In section 4 we reduce the degenerate wave equation to hyperbolic systems and balance laws $2 \times 2$ and $3 \times 3$ of the first order. As to our opinion, using first-order partial differential equations in optimization and control problems is preferable.

In section 5 we shortly discuss numerical methods for obtaining weak solutions to well-posed initial boundary value problems for the degenerate wave equation and degenerate hyperbolic systems and balance laws $2 \times 2$ and $3 \times 3$.

In section 6 we present numerical weak solutions to well-posed initial boundary value problems for the wave equation using regularization.

In section 7 we give in brief our observations on the subject.

The article is a preparatory and preliminary publication when preparing for solving optimization and control problems for the degenerate wave equation.

\section{Pre-formulation of IBVPs for the wave equation}

In the current study our concern relates to initial boundary value problems in space-time rectangle $[0, T] \times[-l,+l]$ for the following linear inhomogeneous wave equation of the second order

$$
\frac{\partial^{2} u}{\partial t^{2}}-\frac{\partial}{\partial x}\left(a \frac{\partial u}{\partial x}\right)=g,
$$

where $t, x$ are the independent variables, $u(t, x)$ is the dependent variable, $a(x)$ is the only coefficient, $g(t, x, u)$ is a source term playing an essential role in problems of control, but being equal zero in the current study.

We apply the chain rule for the second term of equation (2.1) to obtain the expanded form of the latter as follows 


$$
\frac{\partial^{2} u}{\partial t^{2}}-a \frac{\partial^{2} u}{\partial x^{2}}=a^{\prime} \frac{\partial u}{\partial x}+g=: \Phi,
$$

where $a^{\prime} \equiv a^{\prime}(x)$, that is the prime as usual means the first derivative of $a(x)$, and auxiliary quantity $\Phi$ is introduced for the sake of convenience.

Equation (2.2) is known to be of hyperbolic type provided function $a(x)$ is positive, and the same is true for equation (2.1). A subsegment of segment $[-l,+l]$ where function $a(x)$ vanishes, produces a band in the space-time rectangle where both equations lose their hyperbolicity, or degenerate.

We consider a particular case of losing hyperbolicity of equations (2.1) and (2.2) when function $a(x)$ vanishes in a single point inside segment $[-l,+l]$. Let $a_{0}$ be a constant reference value for continuous and piecewise smooth function $a(x)$ that vanishes in the mid point of segment $[-l,+l]$ following a power law inside a narrow subsegment $[-\delta,+\delta]$ as follows (Fig. 2.1,a)

$$
a(x)= \begin{cases}a_{0}, & \delta \leqslant|x| \leqslant l, \\ a_{1}|x|^{\alpha}, & 0 \leqslant|x| \leqslant \delta,\end{cases}
$$

where $0<\delta<l, \alpha>0, a_{1}=a_{0} \delta^{-\alpha}$. Such a function $a(x)$ is easily replaced with its regular (non-vanishing in any point of segment $[-l,+l]$ ) continuous and piecewise smooth counterpart (Fig. 2.1,b)

$$
a_{\varepsilon}(x)= \begin{cases}a_{0}, & \delta \leqslant|x| \leqslant l, \\ a_{2}|x|^{\alpha}+\varepsilon, & 0 \leqslant|x| \leqslant \delta,\end{cases}
$$

where $a_{2}=\left(a_{0}-\varepsilon\right) \delta^{-\alpha}$ and $\varepsilon$ is the regularization parameter, to avoid the degeneracy of equations (2.1) and (2.2). The choice of regular function $a_{\varepsilon}(x)$ is not unique, and replacing singular power curve $|x|^{\alpha}$ with another regular one, for example, with $(|x|+\varepsilon)^{\alpha}$, is possible as well.

The formulation of any admissible well-posed initial boundary value problem for both wave equations (2.1) and (2.2), where the singular coefficient function $a(x)$ given by (2.3) is replaced with a regular function $a_{\varepsilon}(x)$ similar to that given by (2.4), i. e. not leading to degeneracy of equations (2.1) and (2.2), is well known. But what happens if the regularization parameter $\varepsilon$ tends to zero (consequently, $\left.a_{\varepsilon}(x) \rightarrow a(x)\right)$, or it is the same as both wave equations (2.1) and (2.2) degenerate? To answer this question we refer to the theory of characteristics.

\section{Characteristic analysis of IBVPs for the wave equation}

In this section we implement the characteristic analysis to wave equation (2.2), where the coefficient function is given by (2.3) and (2.4), following well known standard approaches.

First, we consider a general linear (in the highest derivatives) partial differential equation of the second order in two independent variables 


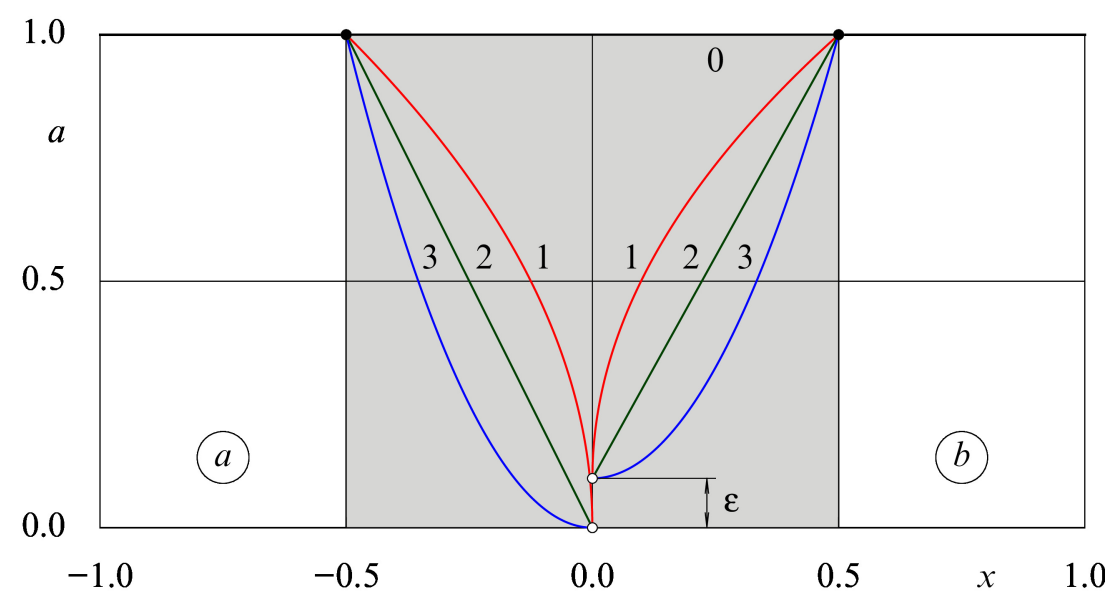

Fig. 2.1. The continuous and piecewise smooth coefficient function $a(x)$ of the wave equation (2.1), (2.2) is defined in segment $[-l,+l]=[-1,+1]$ : vanishing in the mid point $x=0$ of segment (2.3) and referred to as singular $(a)$ and non-vanishing in any point of segment due to the regularization parameter $\varepsilon(2.4)$ and referred to as regular $(b)$; the value of exponent $\alpha$ in power law $|x|^{\alpha}$ inside subsegment $[-\delta,+\delta](2.3),(2.4)$ is chosen to be equal to $0,5,1,2$ (curves marked with 1,2 , and 3 respectively); the constant reference value $a_{0}=1$ is shown as straight line marked with 0 . Subsegment $[-\delta,+\delta]=[-0.5,+0.5]$ is chosen wide for clarity

$$
a_{1,1} p_{1,1}+2 a_{1,2} p_{1,2}+a_{2,2} p_{2,2}=\Psi\left(x_{1}, x_{2}, w, p_{1}, p_{2}\right),
$$

where $x_{1}, x_{2}$ are the independent variables, $w\left(x_{1}, x_{2}\right)$ is the dependent variable, $p_{\kappa}$ are the first derivatives of $w$ with respect to $x_{\kappa}, p_{\iota, \kappa}$ are the second derivatives of $w$ with respect to $x_{\iota}, x_{\kappa}, a_{\iota, \kappa}\left(x_{1}, x_{2}\right)$ are the coefficients of the second derivatives, $\iota, \kappa=1,2,3$.

Second, we consider the following linear algebraic system with respect to the second partial derivatives of function $u$

$$
\left(\begin{array}{ccc}
a_{1,1} & 2 a_{1,2} & a_{2,2} \\
\mathrm{~d} x_{1} & \mathrm{~d} x_{2} & 0 \\
0 & \mathrm{~d} x_{1} & \mathrm{~d} x_{2}
\end{array}\right)\left(\begin{array}{c}
p_{1,1} \\
p_{1,2} \\
p_{2,2}
\end{array}\right)=\left(\begin{array}{c}
\Psi \\
\mathrm{d} p_{1} \\
\mathrm{~d} p_{2}
\end{array}\right),
$$

appearing in the theory of characteristics when treating the Cauchy problem for equation (3.1) along a curve, small segment of which is given by the longitudinal displacements $\mathrm{d} x_{1}$ and $x_{2}$.

The above system has non-trivial and non-unique solutions iff the rank of the matrix of the system equals the rank of the extended matrix of the system, that is the following condition holds 


$$
\operatorname{rank}\left(\begin{array}{cccc}
a_{1,1} & 2 a_{1,2} & a_{2,2} & \Psi \\
\mathrm{d} x_{1} & \mathrm{~d} x_{2} & 0 & \mathrm{~d} p_{1} \\
0 & \mathrm{~d} x_{1} & \mathrm{~d} x_{2} & \mathrm{~d} p_{2}
\end{array}\right)=\operatorname{rank}\left(\begin{array}{ccc}
a_{1,1} & 2 a_{1,2} & a_{2,2} \\
\mathrm{~d} x_{1} & \mathrm{~d} x_{2} & 0 \\
0 & \mathrm{~d} x_{1} & \mathrm{~d} x_{2}
\end{array}\right) .
$$

The above condition determines the characteristic curve of equation (3.1).

For wave equation (2.2) we have: $x_{1}=t, x_{2}=x, a_{1,1}=1, a_{1,2}=0, a_{2,2}=-a$, $p_{1}=\frac{\partial u}{\partial t}, p_{2}=\frac{\partial u}{\partial x}, \Psi=\Phi=a^{\prime} v_{3}+g$, and condition (3.3) reads

$$
\operatorname{rank}\left(\begin{array}{cccc}
1 & 0 & -a & \Phi \\
\mathrm{d} t & \mathrm{~d} x & 0 & \mathrm{~d} p_{1} \\
0 & \mathrm{~d} t & \mathrm{~d} x & \mathrm{~d} p_{2}
\end{array}\right)=2,
$$

where by $a$ it is meant function $a(x)(2.3)$ or function $a_{\varepsilon}(x)(2.4)$.

$$
\begin{aligned}
& \Delta_{0}=\operatorname{det}\left|\begin{array}{ccc}
1 & 0 & -a \\
\mathrm{~d} t & \mathrm{~d} x & 0 \\
0 & \mathrm{~d} t & \mathrm{~d} x
\end{array}\right|=\mathrm{d} x \mathrm{~d} x-a \mathrm{~d} t \mathrm{~d} t=0, \\
& \Delta_{1}=\operatorname{det}\left|\begin{array}{rrr}
0 & -a & \Phi \\
\mathrm{d} x & 0 & \mathrm{~d} p_{1} \\
\mathrm{~d} t & \mathrm{~d} x & \mathrm{~d} p_{2}
\end{array}\right|=\Phi \mathrm{d} x \mathrm{~d} x-a \mathrm{~d} t \mathrm{~d} p_{1}+a \mathrm{~d} x \mathrm{~d} p_{2}=0, \\
& \Delta_{2}=\operatorname{det}\left|\begin{array}{ccc}
1 & -a & \Phi \\
\mathrm{d} t & 0 & \mathrm{~d} p_{1} \\
0 & \mathrm{~d} x & \mathrm{~d} p_{2}
\end{array}\right|=\Phi \mathrm{d} t \mathrm{~d} x-\mathrm{d} x \mathrm{~d} p_{1}+a \mathrm{~d} t \mathrm{~d} p_{2}=0, \\
& \Delta_{3}=\operatorname{det}\left|\begin{array}{ccc}
1 & 0 & \Phi \\
\mathrm{d} t & \mathrm{~d} x & \mathrm{~d} p_{1} \\
0 & \mathrm{~d} t & \mathrm{~d} p_{2}
\end{array}\right|=\Phi \mathrm{d} t \mathrm{~d} t-\mathrm{d} t \mathrm{~d} p_{1}+\mathrm{d} x \mathrm{~d} p_{2}=0 .
\end{aligned}
$$

Determinant $\Delta_{0}$ gives the equation of characteristic curves which splits into the equations of two independent branches

$$
\mathrm{d} x=\mp \sqrt{a} \mathrm{~d} t,
$$

usually referred to as the equations of the $\mp$-(families of) characteristics. 
Replacing $\mathrm{d} x$ in determinants $\Delta_{1}, \Delta_{2}$, and $\Delta_{3}$ with $\mp \sqrt{a} \mathrm{~d} t$ (3.5) we find that the following two equalities hold

$$
\Phi \mathrm{d} t-\mathrm{d} p_{1} \pm \sqrt{a} \mathrm{~d} p_{2}=0,
$$

usually referred to as the relations along the $\mp$-(families of) characteristics.

We assign three different values of the exponent $\alpha$ in (2.3) and (2.4) and find the characteristic curves of both families passing through given points $\left(\mp \delta, t_{0}\right)$.

1) Let $\alpha=0,5$, then integration of differential equations (3.5) gives both families as (case $I_{0}$ )

$$
t=t_{0} \mp \frac{4}{3 \sqrt{a_{1}}}\left(\delta^{\frac{3}{4}}-|x|^{\frac{3}{4}}\right), \quad x \in(0, \delta] .
$$

If the regularization parameter $\varepsilon$ is not equal to zero, then integration of differential equations (3.6) of both families gives (case $I_{\varepsilon}$ )

$$
t=t_{0} \pm \frac{4}{3 a_{2}^{2}}\left[\sqrt{a_{2} \sqrt{\delta}+\varepsilon}\left(a_{2} \sqrt{\delta}-2 \varepsilon\right)-\sqrt{a_{2} \sqrt{|x|}+\varepsilon}\left(a_{2} \sqrt{|x|}-2 \varepsilon\right)\right]
$$

where $x \in[0, \delta]$.

Characteristic curves (3.7) and (3.8) are shown in Fig. 3.2, 1, $a$ and Fig. 3.2, 1, b respectively.

2) Let $\alpha=1$, then integration of differential equations (3.5) gives both families as $\left(\right.$ case $\left.I I_{0}\right)$

$$
t=t_{0} \mp \frac{2}{\sqrt{a_{1}}}[\sqrt{\delta}-\sqrt{|x|}], \quad|x| \in(0, \delta] .
$$

If the regularization parameter $\varepsilon$ is not equal to zero, then integration of differential equations (3.6) of both families gives (case $I I_{\varepsilon}$ )

$$
t=t_{0} \pm \frac{2}{a_{2}}\left[\sqrt{a_{2} \delta+\varepsilon}-\sqrt{a_{2}|x|+\varepsilon}\right], \quad|x| \in[0, \delta] .
$$

The characteristic curves (3.9) and (3.10) are shown in Fig. 3.2,2, $a$ and Fig. $3.2,2, b$ respectively.

3) Let $\alpha=2$, then then integration of differential equations (3.5) gives both families as (case $\left.I I I_{0}\right)$

$$
t=t_{0} \mp \frac{1}{\sqrt{a_{1}}}[\ln \delta-\ln |x|], \quad|x| \in(0, \delta] .
$$

If the regularization parameter $\varepsilon$ is not equal to zero, then integration of differential equations (3.6) of both families gives (case $I I I_{\varepsilon}$ )

$$
t=t_{0} \pm \frac{1}{a_{2}}\left[\ln \left(\sqrt{a_{2} \delta^{2}+\varepsilon}+\sqrt{a_{2} \delta^{2}}\right)-\ln \left(\sqrt{a_{2} x^{2}+\varepsilon}+\sqrt{a_{2} x^{2}}\right)\right]
$$


where $|x| \in[0, \delta]$.

The characteristic curves (3.11) and (3.12) are shown in Fig. 3.2,(3) $a$ and Fig. $3.2,(3) b$ respectively.

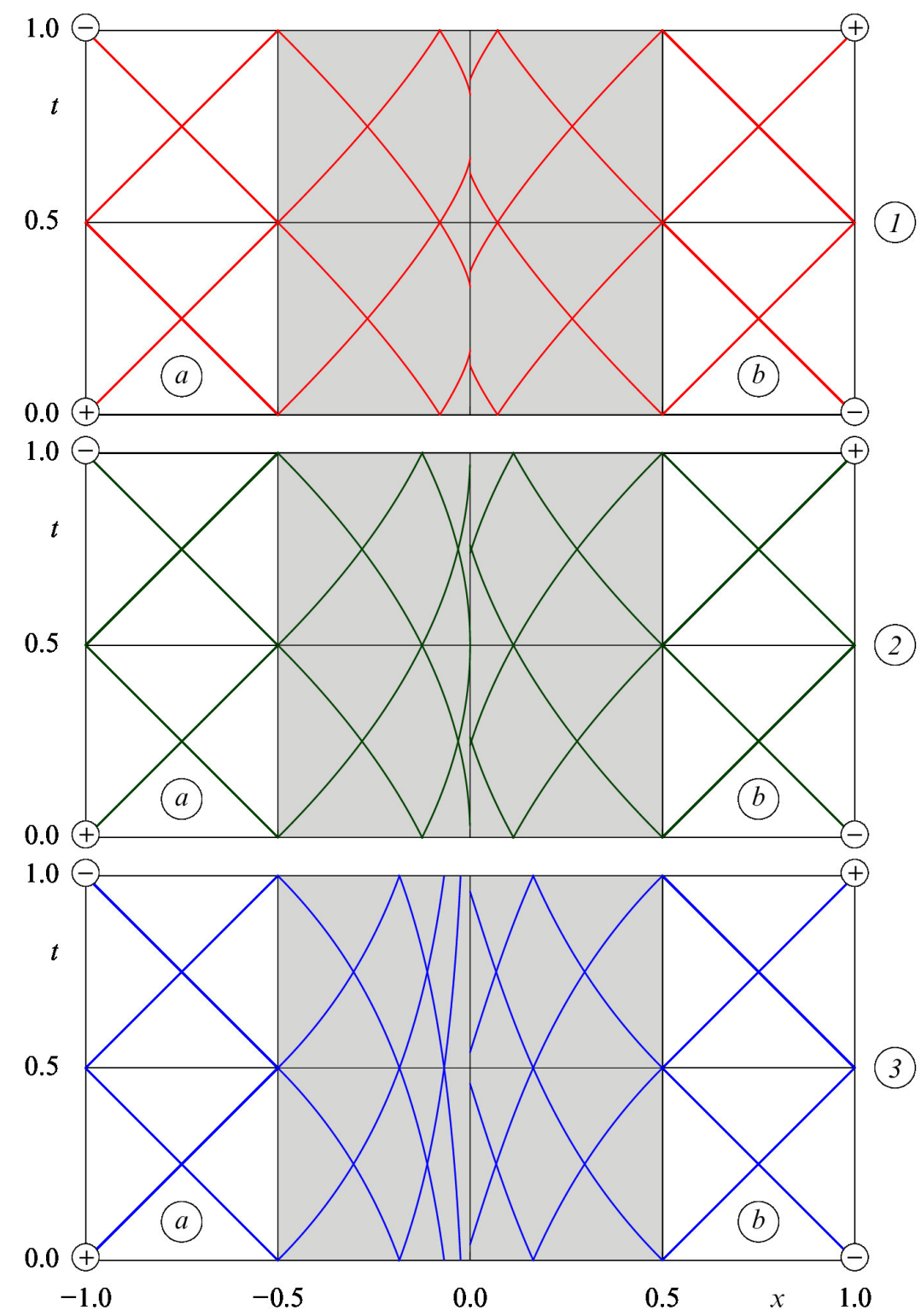

Fig. 3.2. $\mp$-families of characteristics for the wave equation (2.1), (2.2) depending on the value of exponent $\alpha$ in power law $|x|^{\alpha}$ inside subsegment $[-\delta,+\delta]=[-0.5,+0.5](2.3),(2.4): \alpha=0.5(1)$, $\alpha=1(2), \alpha=2(3)$, without regularization $(a)$ and with regularization $(b)$

From Fig. 3.2 it is evident that:

1. On straight line $x=0$, wave equation (2.2) degenerates into the following 
partial differential equation of parabolic type

$$
\frac{\partial^{2} u}{\partial t^{2}}=a^{\prime} \frac{\partial u}{\partial x}+g
$$

in cases $I_{0}(0<\alpha<1)$ and $I I_{0}(\alpha=1)$ and into the following ordinary differential equation

$$
\frac{\partial^{2} u}{\partial t^{2}}=g
$$

in case $I I I_{0}(\alpha>1)$.

In case $I_{0}$ on straight line $x=0$ function $a(x)$ vanishes, whereas its first derivative $a^{\prime}(x)$ becomes unbounded. This means that if wave equation (2.2) has a bounded solution, then the first term on its right-hand side is also bounded, and the following estimates hold

$$
a^{\prime} \frac{\partial u}{\partial x} \sim \mathscr{O}(1), \quad \frac{\partial u}{\partial x} \sim \frac{1}{a^{\prime}} \sim \mathscr{O}(\sqrt{x}), \quad u(x) \sim \mathscr{O}\left(x^{3 / 2}\right) .
$$

2. In cases $I_{0}, I I_{0}, I I I_{0}$ no characteristic of wave equation (2.2) crosses straight line $x=0$ from the left to the right and vice versa, and this means that the solutions of the equation in left and right subregions of space-time rectangle can be found quite independently of each other.

3. In cases $I_{0, \varepsilon}, I I_{0, \varepsilon}$ and $I I I_{\varepsilon}$ at each point of straight line $x=0$ there are one characteristic incoming from the left and one incoming from the right, as well as one characteristic outgoing to the left and one outgoing to the right. From this we conclude that on straight line $x=0$ one boundary condition needs to be posed.

4. In case $I I I_{0}$ at each point of straight line $x=0$ there is no characteristic incoming from the left and incoming from the right, as well as no characteristic outgoing to the left and outgoing to the right. From this we conclude that on straight line $x=0$ no boundary condition needs to be posed, and value $u(t, 0)$ can be obtained directly from second order ordinary differential equation (3.14).

\section{Pre-formulation of IBVPs for the derived hyperbolic systems}

In the current section we reduce wave equations (2.1) and (2.2) to systems of linear partial differential equations of the first order.

We start our reducing wave equation (2.2) from the following evident operator identity

$$
\begin{aligned}
\frac{\partial^{2}}{\partial t^{2}}-a \frac{\partial^{2}}{\partial x^{2}} & =\left(\frac{\partial}{\partial t}-\sqrt{a} \frac{\partial}{\partial x}\right)\left(\frac{\partial}{\partial t}+\sqrt{a} \frac{\partial}{\partial x}\right)+\frac{1}{2} a^{\prime} \frac{\partial}{\partial x}= \\
& =\left(\frac{\partial}{\partial t}+\sqrt{a} \frac{\partial}{\partial x}\right)\left(\frac{\partial}{\partial t}-\sqrt{a} \frac{\partial}{\partial x}\right)+\frac{1}{2} a^{\prime} \frac{\partial}{\partial x}
\end{aligned}
$$

and then easily rewrite the wave equation as the following two systems 


$$
\begin{aligned}
& \left\{\begin{array}{l}
\frac{\partial u_{1}}{\partial t}+\sqrt{a} \frac{\partial u_{1}}{\partial x}-u_{2}=0 \\
\frac{\partial u_{2}}{\partial t}-\frac{a^{\prime}}{2} \frac{\partial u_{1}}{\partial x}-\sqrt{a} \frac{\partial u_{2}}{\partial x}=g
\end{array}\right. \\
& \left\{\begin{array}{l}
\frac{\partial v_{1}}{\partial t}-\sqrt{a} \frac{\partial v_{1}}{\partial x}-v_{2}=0 \\
\frac{\partial v_{2}}{\partial t}-\frac{a^{\prime}}{2} \frac{\partial v_{1}}{\partial x}+\sqrt{a} \frac{\partial v_{2}}{\partial x}=g
\end{array}\right.
\end{aligned}
$$

where $u_{1}(t, x)=v_{1}(t, x)=u(t, x)$, whereas functions $u_{2}(t, x)$ and $v_{2}(t, x)$ are fully determined by the first equations of the above systems.

Both systems can be presented in matrix form, for example the former system reads

$$
\frac{\partial \mathbf{U}}{\partial t}+\mathrm{A} \frac{\partial \mathbf{U}}{\partial x}+\mathrm{A}_{\star} \mathbf{U}=\mathbf{G}_{o}
$$

where $\mathbf{U}(t, x)$ and $\mathbf{G}_{o}(t, x, \mathbf{U})$ are respectively the state and the right hand side matrix-columns, $\mathrm{A}(x)$ and $\mathrm{A}_{\star}$ are quadratic coefficient matrices as follows

$$
\mathbf{U}=\left(\begin{array}{l}
u_{1} \\
u_{2}
\end{array}\right), \quad \mathbf{G}_{o}=\left(\begin{array}{l}
g \\
0
\end{array}\right), \quad \mathbf{A}=\left(\begin{array}{cc}
+\sqrt{a} & 0 \\
-\frac{1}{2} a^{\prime} & -\sqrt{a}
\end{array}\right), \quad \mathrm{A}_{\star}=\left(\begin{array}{cc}
0 & -1 \\
0 & 0
\end{array}\right) .
$$

Matrix A has two real and distinct eigenvalues $\lambda_{1,2}=\mp \sqrt{a}$ and a complete set of right (in columns of matrix $R$ ) and left (in rows of matrix $L$ ) eigenvectors

$$
\mathrm{R}=\mathrm{L}^{-1}=\left(\begin{array}{cc}
0 & 1 \\
1 & -\frac{1}{2}(\sqrt{a})^{\prime}
\end{array}\right), \quad \mathrm{L}=\mathrm{R}^{-1}=\left(\begin{array}{cc}
+\frac{1}{2}(\sqrt{a})^{\prime} & 1 \\
1 & 0
\end{array}\right),
$$

hence, system (4.3) is strictly hyperbolic. Matrices $\mathrm{R}$ and $\mathrm{L}$ diagonalize matrix $\mathrm{A}$

$$
\Lambda=\mathrm{LAR}=\left(\begin{array}{cc}
-\sqrt{a} & 0 \\
0 & +\sqrt{a}
\end{array}\right)=\operatorname{diag}(-\sqrt{a},+\sqrt{a})
$$

and produce splitting matrix $A$ into sum

$$
A=A^{-}+A^{+}
$$

of non-positive and non-negative definite matrices

$$
\mathrm{A}^{-}=\mathrm{R} \Lambda^{-} \mathrm{L}=\left(\begin{array}{cc}
0 & 0 \\
-\frac{1}{4} a^{\prime} & -\sqrt{a}
\end{array}\right), \quad \mathrm{A}^{+}=\mathrm{R} \Lambda^{+} \mathrm{L}=\left(\begin{array}{cc}
+\sqrt{a} & 0 \\
-\frac{1}{4} a^{\prime} & 0
\end{array}\right)
$$


where

$$
\Lambda^{-}=\operatorname{diag}(-\sqrt{a}, 0), \quad \Lambda^{+}=\operatorname{diag}(0,+\sqrt{a}), \quad \Lambda^{-}+\Lambda^{+}=\Lambda .
$$

To make weak solutions of hyperbolic system (4.3) admissible we rewrite the former as a vector balance law

$$
\frac{\partial \mathbf{U}}{\partial t}+\frac{\partial \mathbf{F}}{\partial x}=\mathbf{G}
$$

where the state $\mathbf{U}(t, x)$ is the same as in (4.3), whereas flux $\mathbf{F}(x, \mathbf{U})=\mathrm{A}(x) \mathbf{U}(t, x)$ and source term $\mathbf{G}(t, x, \mathbf{U})$ are

$\mathbf{F}=\left(\begin{array}{c}\sqrt{a} u_{1} \\ -\frac{1}{2} a^{\prime} u_{1}-\sqrt{a} u_{2}\end{array}\right), \quad \mathbf{G}=\mathbf{G}_{o}+\mathrm{A}_{o} \mathbf{U}, \quad \mathrm{A}_{o}=\mathrm{A}^{\prime}-\mathrm{A}_{\star}=\left(\begin{array}{cc}+(\sqrt{a})^{\prime} & 1 \\ -\frac{1}{2} a^{\prime \prime} & -(\sqrt{a})^{\prime}\end{array}\right)$,

matrix A being the Jacobian of the flux. Splitting (4.4), (4.5) of Jacobian A produces evident splitting of the flux as

$$
\mathbf{F}=\mathrm{A} \mathbf{U}=\mathrm{A}^{-} \mathbf{U}+\mathrm{A}^{+} \mathbf{U}=\mathbf{F}^{-}+\mathbf{F}^{+} .
$$

Another way to reduce wave equation (2.2) is introducing new dependent variables $u_{1}=u, u_{2}=\frac{\partial u}{\partial t}, u_{3}=\frac{\partial u}{\partial x}$, then the wave equation is rewritten as the following system

$$
\frac{\partial \mathbf{U}}{\partial t}+\mathrm{A} \frac{\partial \mathbf{U}}{\partial x}+\mathrm{A}_{\star} \mathbf{U}=\mathbf{G}_{o}
$$

where $\mathbf{U}(t, x)$ and $\mathbf{G}_{o}(t, x, \mathbf{U})$ are respectively the state and the right hand side matrix-columns, $\mathrm{A}(x)$ and $\mathrm{A}_{\star}(x)$ are quadratic coefficient matrices as follows

$$
\mathbf{U}=\left(\begin{array}{c}
u_{1} \\
u_{2} \\
u_{3}
\end{array}\right), \quad \mathrm{A}=\left(\begin{array}{rrr}
0 & 0 & 0 \\
0 & 0 & -a \\
0 & -1 & 0
\end{array}\right), \quad \mathrm{A}_{\star}=\left(\begin{array}{rrr}
0 & -1 & 0 \\
0 & 0 & -a^{\prime} \\
0 & 0 & 0
\end{array}\right), \quad \mathbf{G}=\left(\begin{array}{l}
0 \\
g \\
0
\end{array}\right)
$$

Matrix $A$ has three real and distinct eigenvalues $\lambda_{1,2,3}=-\sqrt{a}, 0,+\sqrt{a}$, and a complete set of right and left eigenvectors

$$
\mathrm{R}=\left(\begin{array}{ccc}
0 & 1 & 0 \\
+\sqrt{a} & 0 & -\sqrt{a} \\
1 & 0 & 1
\end{array}\right), \quad \mathrm{L}=\mathrm{R}^{-1}=\left(\begin{array}{ccc}
0 & +\frac{1}{2 \sqrt{a}} & \frac{1}{2} \\
1 & 0 & 0 \\
0 & -\frac{1}{2 \sqrt{a}} & \frac{1}{2}
\end{array}\right)
$$


hence, system (4.8) is strictly hyperbolic.

System (4.8) is easily rewritten to a vector balance law

$$
\frac{\partial \mathbf{U}}{\partial t}+\frac{\partial \mathbf{F}}{\partial x}=\mathbf{G},
$$

where the state $\mathbf{U}(t, x)$ is the same as in (4.8), whereas flux $\mathbf{F}(x, \mathbf{U})=\mathrm{A}(x) \mathbf{U}(t, x)$ and source term $\mathbf{G}(t, x, \mathbf{U})$ are

$$
\mathbf{F}=\left(\begin{array}{r}
0 \\
-a u_{3} \\
-u_{2}
\end{array}\right), \quad \mathbf{G}=\mathbf{G}_{o}+\mathrm{A}_{o} \mathbf{U}, \quad \mathrm{A}_{o}=\mathrm{A}^{\prime}-\mathrm{A}_{\star}=\left(\begin{array}{ccc}
0 & 1 & 0 \\
0 & 0 & 0 \\
0 & 0 & 0
\end{array}\right) \text {. }
$$

Matrices $\mathrm{R}$ and $\mathrm{L}$ diagonalize matrix $\mathrm{A}$

$$
\Lambda=\mathrm{LAR}=\left(\begin{array}{ccc}
-\sqrt{a} & 0 & 0 \\
0 & 0 & 0 \\
0 & 0 & +\sqrt{a}
\end{array}\right)=\operatorname{diag}(-\sqrt{a}, 0,+\sqrt{a}),
$$

and produce splitting matrix $A$

$$
A=A^{-}+A^{+}
$$

and splitting flux

$$
\mathbf{F}=\mathrm{A} \mathbf{U}=\mathrm{A}^{-} \mathbf{U}+\mathrm{A}^{+} \mathbf{U}=\mathbf{F}^{-}+\mathbf{F}^{+}
$$

where

$$
\begin{gathered}
\mathrm{A}^{\mp}=\mathrm{R} \Lambda^{\mp} \mathrm{L}=\left(\begin{array}{ccc}
0 & 0 & 0 \\
0 & \mp \frac{1}{2 \sqrt{a}} & -\frac{1}{2} a \\
0 & -\frac{1}{2} & \mp \frac{1}{2} a
\end{array}\right), \\
\Lambda=\Lambda^{-}+\Lambda^{+}=\operatorname{diag}(-\sqrt{a}, 0,0)+\operatorname{diag}(0,0,+\sqrt{a}), \\
\mathbf{F}^{\mp}=\mathrm{A}^{\mp} \mathbf{U}=\mp \frac{1}{2} \sqrt{a}\left(u_{2} \pm \sqrt{a} u_{3}\right)\left(\begin{array}{c}
0 \\
\sqrt{a} \\
\pm 1
\end{array}\right) .
\end{gathered}
$$


Flux splitting (4.4), (4.5), (4.7), and (4.10), (4.11), (4.12), (4.13) is used in section 5 , where discrete models of wave propagation are considered.

Presenting wave equations (2.2) and (2.1) as hyperbolic systems and balance laws makes it possible to well-pose initial boundary value problems using the theory of characteristics in a quite formal notation of Chakravarthy [2]

$$
\begin{gathered}
\mathrm{L}_{1} \frac{\partial \mathbf{U}}{\partial t}+\mathrm{L}_{2} \mathrm{~A} \frac{\partial \mathbf{U}}{\partial x}+\mathrm{L}_{2} \mathrm{~A}_{\star} \mathbf{U}=\mathrm{L}_{2} \mathbf{G}_{o} \\
\mathrm{~L}_{1} \frac{\partial \mathbf{U}}{\partial t}+\mathrm{L}_{2} \frac{\partial \mathbf{F}}{\partial x}=\mathrm{L}_{2} \mathbf{G}
\end{gathered}
$$

where matrices $L_{1}$ and $L_{2}$ are derived from matrix $L$ of the left eigenvectors and proper boundary conditions. Since matrix $L_{1}$ is non-degenerate, the above coupled systems can be rewritten as

$$
\begin{gathered}
\frac{\partial \mathbf{U}}{\partial t}+\mathrm{L}_{3} \mathrm{~A} \frac{\partial \mathbf{U}}{\partial x}+\mathrm{L}_{3} \mathrm{~A}_{\star} \mathbf{U}=\mathrm{L}_{3} \mathbf{G}_{o}, \\
\frac{\partial \mathbf{U}}{\partial t}+\mathrm{L}_{3} \frac{\partial \mathbf{F}}{\partial x}=\mathrm{L}_{3} \mathbf{G},
\end{gathered}
$$

where $\mathrm{L}_{3}=\mathrm{L}_{1}^{-1} \mathrm{~L}_{2}$.

We also note that balance law (4.6) allows two running waves, while balance law (4.9) allows two running and one standing waves.

\section{Discrete formulation of the former problems}

In order to develop discrete models of problems posed in sections 2,3 , and 4 , we introduce a uniform structured grid in space-time rectangle $[0, T] \times[-l,+l]$, as shown in Fig. 5.3.

Then we apply the Green theorem to balance laws (4.6) and (4.9) over the boundary (irregular) and the interior (regular) cells $\omega_{k}^{n}$ of the grid

$$
\oint_{\gamma_{k}^{n}} \mathbf{U} \mathrm{d} x-\mathbf{F} \mathrm{d} t=\iint_{\omega_{k}^{n}} \mathbf{G} \mathrm{d} t \mathrm{~d} x
$$

where integration along the boundaries $\gamma_{k}^{n}$ of the cells is performed counterclockwise as shown in Fig. 5.4.

Evaluating the line integrals over the lateral interfaces of the cells $\omega_{k}^{n}$ (i. e. over the vertical segments $x=x_{k \mp h}, t^{n} \leqslant t \leqslant t^{n+1}, 1<k<K, 1 \leqslant n<N$, of the boundaries $\gamma_{k}^{n}$ ) using the values of the fluxes at the lower time levels $n$ yields to the following explicit approximation of balance law (4.6) 


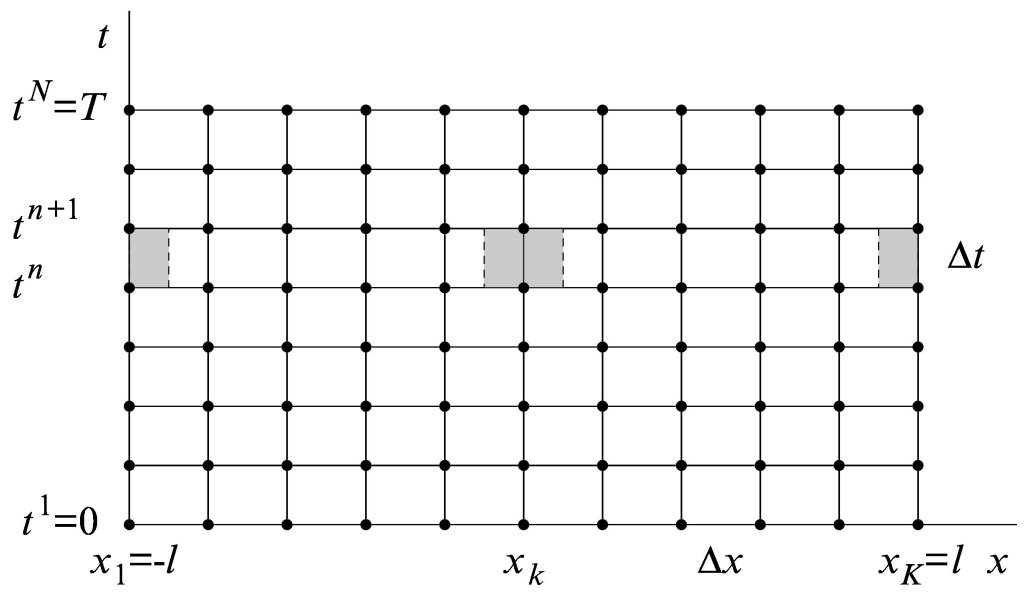

Fig. 5.3. A structured grid in space-time rectangle $[0, T] \times[-l,+l]$ is composed of $N \times K$ nodes with coordinates $x_{k}^{n}$. Using the nodes, the rectangle is divided into the grid cells, the boundary and the interior ones (an interior and two boundary ones are shown in gray color, see Fig. 5.4)

(a)

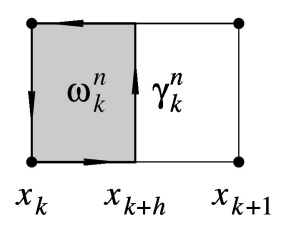

(b)

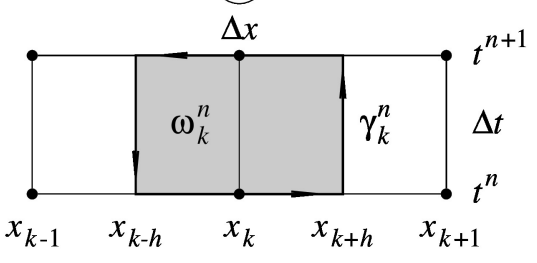

(c)

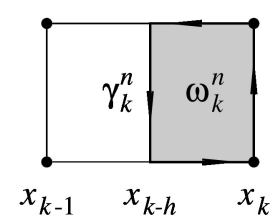

Fig. 5.4. Enlarged view of the boundary $(a, c)$ and interior $(b)$ cells, shown in Fig. 5.3 in gray color: each cell is a region $\omega_{k}^{n}$ with the boundary $\gamma_{k}^{n}$ composed of four straight segments; integration along the boundaries $\gamma_{k}^{n}$ of the cells $\omega_{k}^{n}$ in the Green theorem (5.1) is performed counterclockwise

$$
\begin{aligned}
& \left(\mathbf{U}_{k}^{n+1}-\mathbf{U}_{k}^{n}\right) \frac{\Delta x}{2}+\left(\mathbf{F}_{k+h}^{n}-\mathbf{F}_{k}^{n}\right) \Delta t=\mathbf{G}_{k}^{n} \Delta t \frac{\Delta x}{2}, \quad 1=k, \\
& \left(\mathbf{U}_{k}^{n+1}-\mathbf{U}_{k}^{n}\right) \Delta x+\left(\mathbf{F}_{k+h}^{n}-\mathbf{F}_{k-h}^{n}\right) \Delta t=\mathbf{G}_{k}^{n} \Delta t \Delta x, \quad 1<k<K, \\
& \left(\mathbf{U}_{k}^{n+1}-\mathbf{U}_{k}^{n}\right) \frac{\Delta x}{2}+\left(\begin{array}{ll}
\mathbf{F}_{k}^{n} & -\mathbf{F}_{k-h}^{n}
\end{array}\right) \Delta t=\mathbf{G}_{k}^{n} \Delta t \frac{\Delta x}{2}, \quad k=K,
\end{aligned}
$$

where notation $h:=\frac{1}{2}$ is used for simple referring to the cell interfaces. A more familiar form of the above explicit approximation is as follows 


$$
\left\{\begin{array}{lr}
\frac{\mathbf{U}_{k}^{n+1}-\mathbf{U}_{k}^{n}}{\Delta t}+\frac{\mathbf{F}_{k+h}^{n}-\mathbf{F}_{k}^{n}}{\Delta x / 2}=\mathbf{G}_{k}^{n}, & 1=k, \\
\frac{\mathbf{U}_{k}^{n+1}-\mathbf{U}_{k}^{n}}{\Delta t}+\frac{\mathbf{F}_{k+h}^{n}-\mathbf{F}_{k-h}^{n}}{\Delta x}=\mathbf{G}_{k}^{n}, & 1<k<K, \\
\frac{\mathbf{U}_{k}^{n+1}-\mathbf{U}_{k}^{n}}{\Delta t}+\frac{\mathbf{F}_{k}^{n}-\mathbf{F}_{k-h}^{n}}{\Delta x / 2}=\mathbf{G}_{k}^{n}, & k=K,
\end{array}\right.
$$

where the fluxes through the cell interfaces are evaluated using: 1) Jacobian A splitting (4.4), (4.5), (4.10), (4.12)

$$
\mathbf{F}_{k \mp h}=\mathbf{F}^{+}\left(\mathbf{U}_{k \mp h}^{-}\right)+\mathbf{F}^{-}\left(\mathbf{U}_{k \mp h}^{+}\right)=\mathrm{A}_{k \mp h}^{+} \mathbf{U}_{k \mp h}^{-}+\mathrm{A}_{k \mp h}^{-} \mathbf{U}_{k \mp h}^{+}
$$

and 2) a limited reconstruction of the states $\mathbf{U}_{k \mp h}^{\mp}$ at the cell interfaces after Kolgan $[5,8]$ and after van Leer $[6,7]$ (the latter usually being referred to as MUSCL approach), to achieve the second order in space variable $x$. The proper boundary formulations (4.17) are approximated similarly to (5.2) as

$$
\begin{cases}\frac{\mathbf{U}_{k}^{n+1}-\mathbf{U}_{k}^{n}}{\Delta t}+\mathrm{L}_{3, k}^{n} \frac{\mathbf{F}_{k+h}^{n}-\mathbf{F}_{k}^{n}}{\Delta x / 2}=\mathrm{L}_{3, k}^{n} \mathbf{G}_{k}^{n}, & k=1, \\ \frac{\mathbf{U}_{k}^{n+1}-\mathbf{U}_{k}^{n}}{\Delta t}+\mathrm{L}_{3, k}^{n} \frac{\mathbf{F}_{k}^{n}-\mathbf{F}_{k-h}^{n}}{\Delta x / 2}=\mathrm{L}_{3, k}^{n} \mathbf{G}_{k}^{n}, & k=K,\end{cases}
$$

and replace the respective approximations in (5.2) not coupled with the boundary conditions.

Approximation (5.2), (5.3), (5.4) is nothing but numerical method of Beam and Warming [11], whereas applying splitting (4.4), (4.5), (4.10), (4.12) to hyperbolic systems (4.3) and (4.8) leads to the split coefficient matrix method of Chakravarthy et. al. [1].

Approximation (5.2), (5.4) being of the first order in time is easily transformed to the second order one. For this purpose we apply the approximation sequentially two times: first to find state $\mathbf{U}^{\overline{n+1}}$ using state $\mathbf{U}^{n+1}$ and second to find state $\mathbf{U}^{\overline{n+1}}$ using state $\mathbf{U}^{\overline{n+1}}$, then the required state $\mathbf{U}^{n+1}$ is obtained by averaging

$$
\mathbf{U}_{k}^{n+1}=\frac{1}{2}\left(\mathbf{U}_{k}^{n}+\mathbf{U}_{k}^{\overline{n+1}}\right), \quad 1 \leqslant k \leqslant K,
$$

similarly to the predictor-corrector method of MacCormack $[9,10]$.

Applying the Green theorem to wave equation (2.1) over the double deck interior cells (such cells are obtained by coupling cells $\omega_{k}^{n}$ and $\omega_{k}^{n+1}$ ) gives the well known three time level approximation for the interior nodes

$$
\frac{u_{k}^{n+1}-2 u_{k}^{n}+u_{k}^{n-1}}{(\Delta t)^{2}}=\frac{a_{k+h} \frac{u_{k+1}^{n}-u_{k}^{n}}{\Delta x}-a_{k-h} \frac{u_{k}^{n}-u_{k-1}^{n}}{\Delta x}}{\Delta x}+g_{k}^{n} .
$$




\section{Numerical solutions to test cases}

In the current section we present the solutions to initial boundary value problems for wave equation (2.1) where coefficient $a$ is given by regular function $a_{\varepsilon}(x)(2.4)$. The initial piece-wise constant conditions are chosen as follows

$$
\begin{aligned}
& u(0, x)=\left\{\begin{array}{ll}
0, & \left|x+\frac{1}{2}\right|>\frac{1}{2} h_{x}, \\
h_{y}, & \left|x+\frac{1}{2}\right| \leqslant \frac{1}{2} h_{x},
\end{array} \quad x \in[-l, 0],\right. \\
& u(0, x)=\left\{\begin{array}{ll}
0, & \left|x-\frac{1}{2}\right|>\frac{1}{2} h_{x}, \\
h_{y}, & \left|x-\frac{1}{2}\right| \leqslant \frac{1}{2} h_{x},
\end{array} \quad x \in[0,+l],\right. \\
& \frac{\partial u(0, x)}{\partial t}=0, \quad x \in[-l,+l],
\end{aligned}
$$

where $h_{x}=0.2, h_{y}=0.5, \delta=0.1$. The boundary conditions are set as

$$
u(t,-l)=u(t,+l)=0 .
$$

Four different values $0,0.5,1$, and 2 are assigned to exponent $\alpha$ in power law (2.3), and the regularization parameter is set to be 0.05 . Time segment $[0, T]$ is taken as $[0,3]$. The number of spatial nodes $K=2001$.

Weak numerical solutions to the problems were obtained using: 1) directly wave equation $(2.1) ; 2$ ) hyperbolic balance law $2 \times 2(4.6)$ and 3 ) hyperbolic balance law $3 \times 3$ (4.9). Comparing the solutions to these initial boundary value problems obtained in three different ways will be given elsewhere, and results obtained for wave equation (2.1) using approximation (5.6) are presented below in Figs 8.58.9 , where solutions for different values of $\alpha$ can be easily recognized, since the are marked the same way as in Figs. 2.1 and 3.2. Spatial-time band $[0,3] \times[0,+1]$ is shown in gray color.

The results obtained prove that the value of the exponent in power law (2.4) affects the solutions of wave equation (2.1) in a non-trivial way.

\section{Conclusions}

The solutions to four initial boundary value problems for wave equation (2.1) obtained in the current study give a preliminary evidence in what way the value of exponent in power law (2.4) affects the solutions. Results obtained for degenerate wave equation (2.1) will be given elsewhere.

\section{Acknowledgements}

The current study was inspired and motivated by Prof. P. I. Kogut of Dnipro National University. Discussion with him of ways of regularization of the degenerate wave equation, formulations of initial boundary value problems for the equation and preliminary results was useful. 

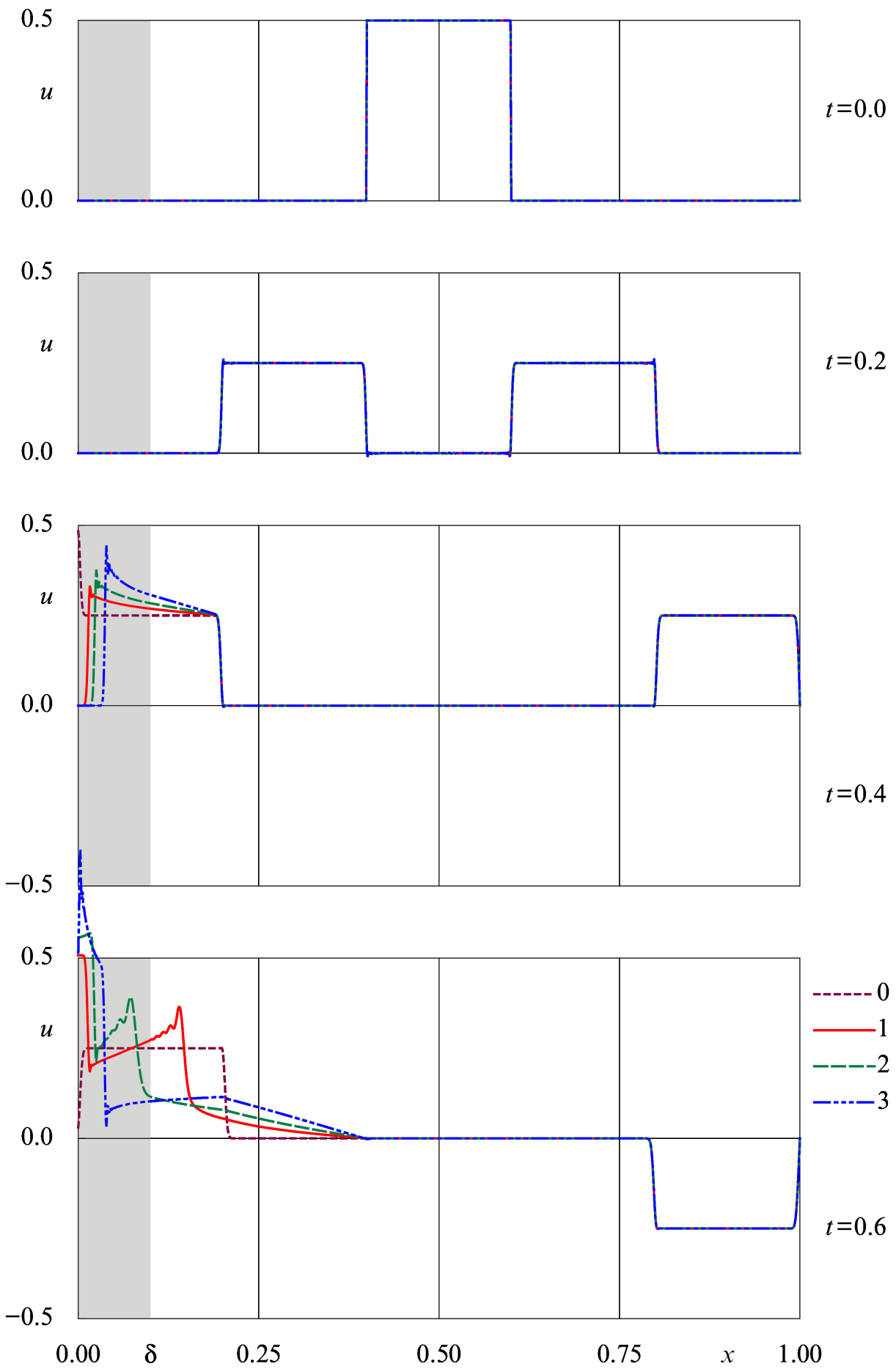

Fig. 8.5. Numerical solutions to four initial boundary value problems for wave equation (2.1) using approximation (5.6) 

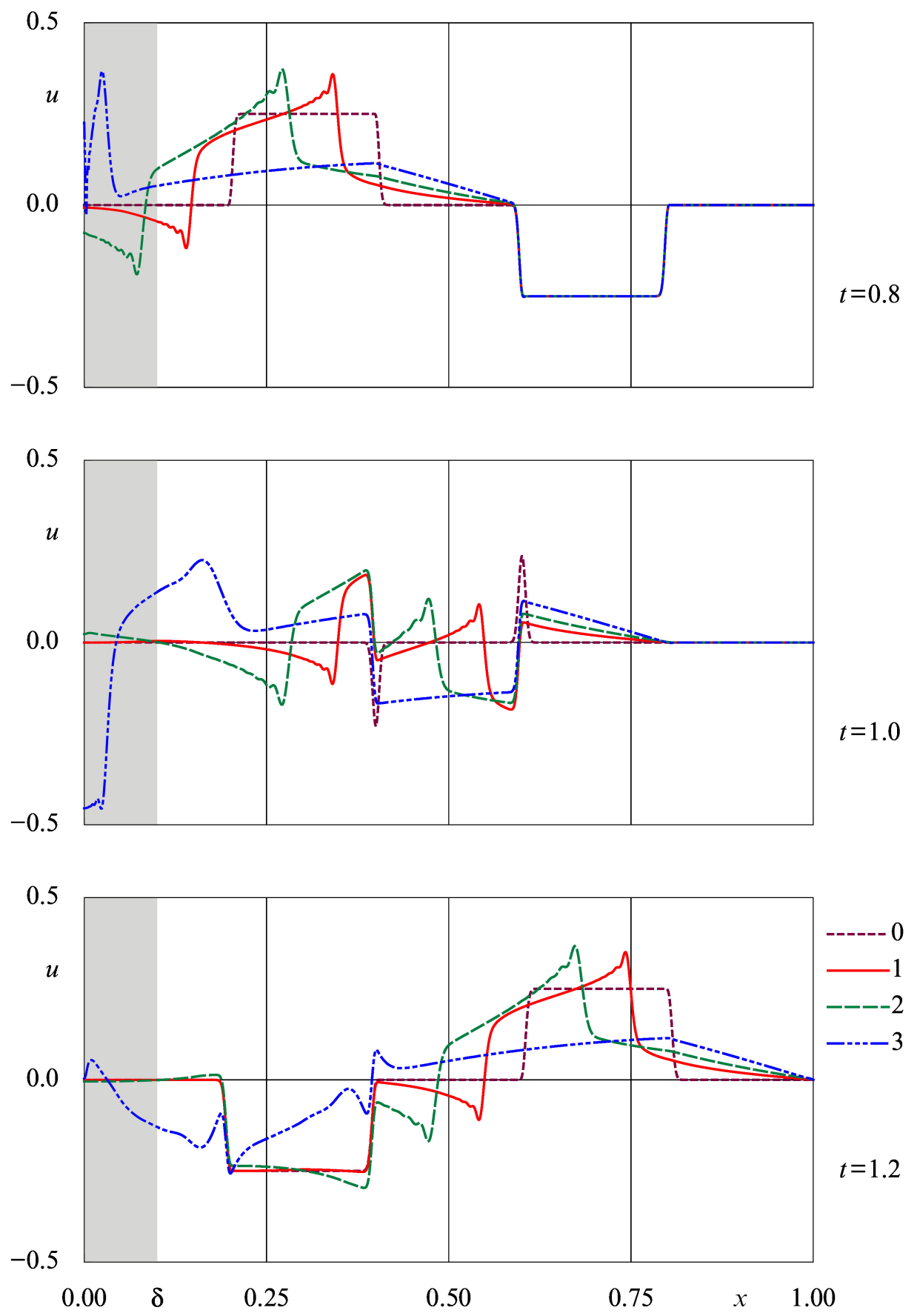

Fig. 8.6. Numerical solutions to four initial boundary value problems for wave equation (2.1) using approximation (5.6) (continuation) 

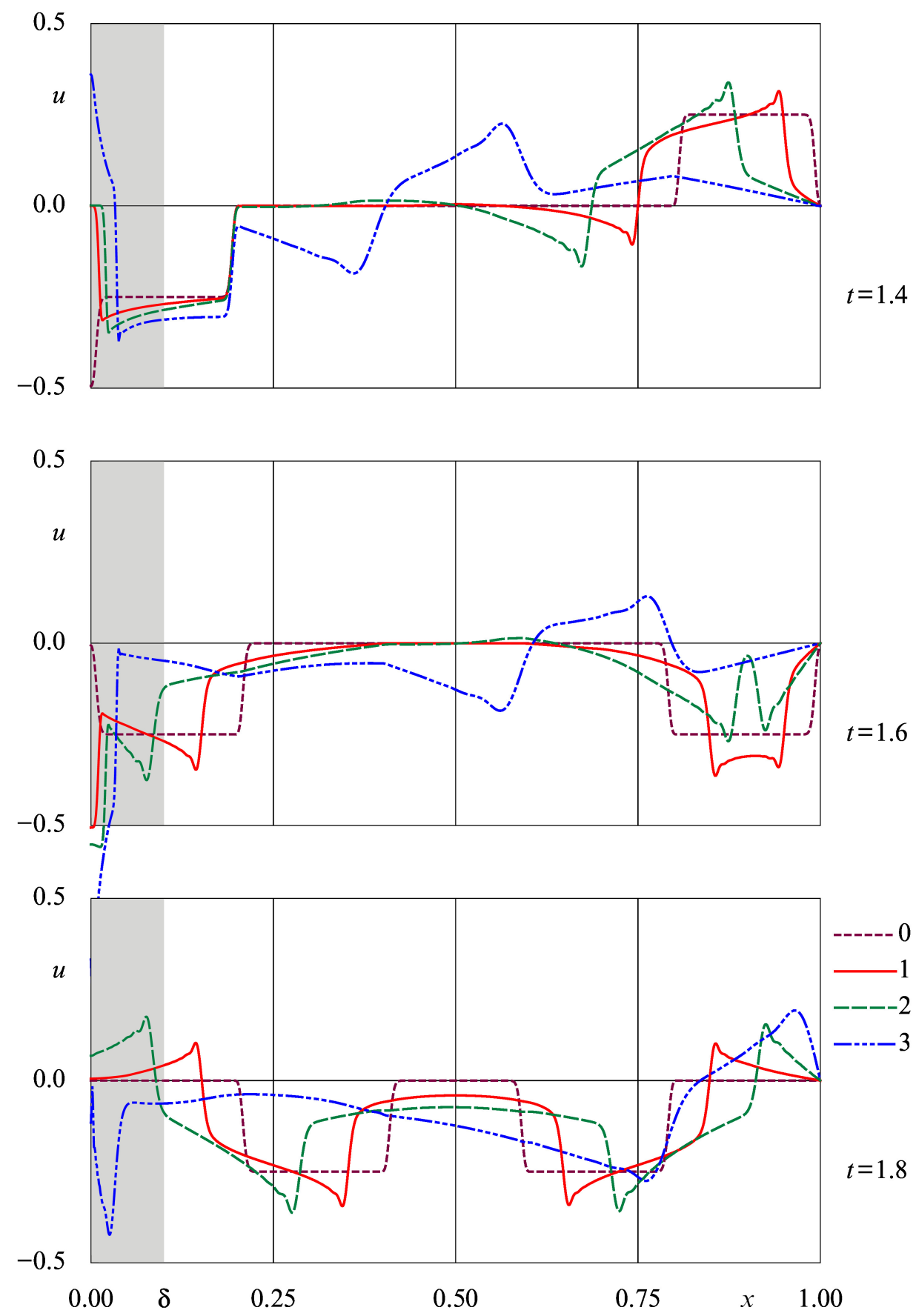

Fig. 8.7. Numerical solutions to four initial boundary value problems for wave equation (2.1) using approximation (5.6) (continuation) 

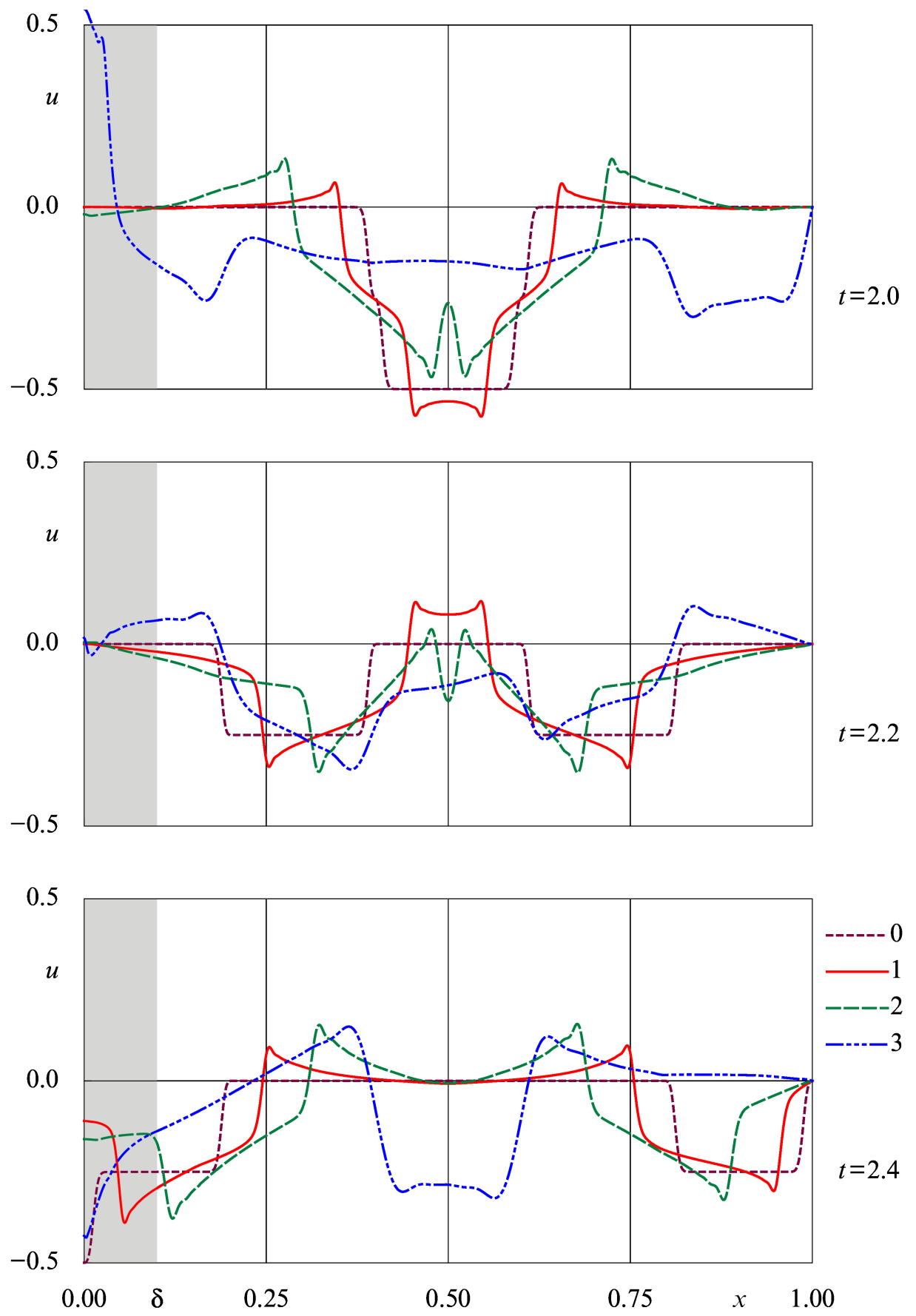

Fig. 8.8. Numerical solutions to four initial boundary value problems for wave equation (2.1) using approximation (5.6) (continuation) 

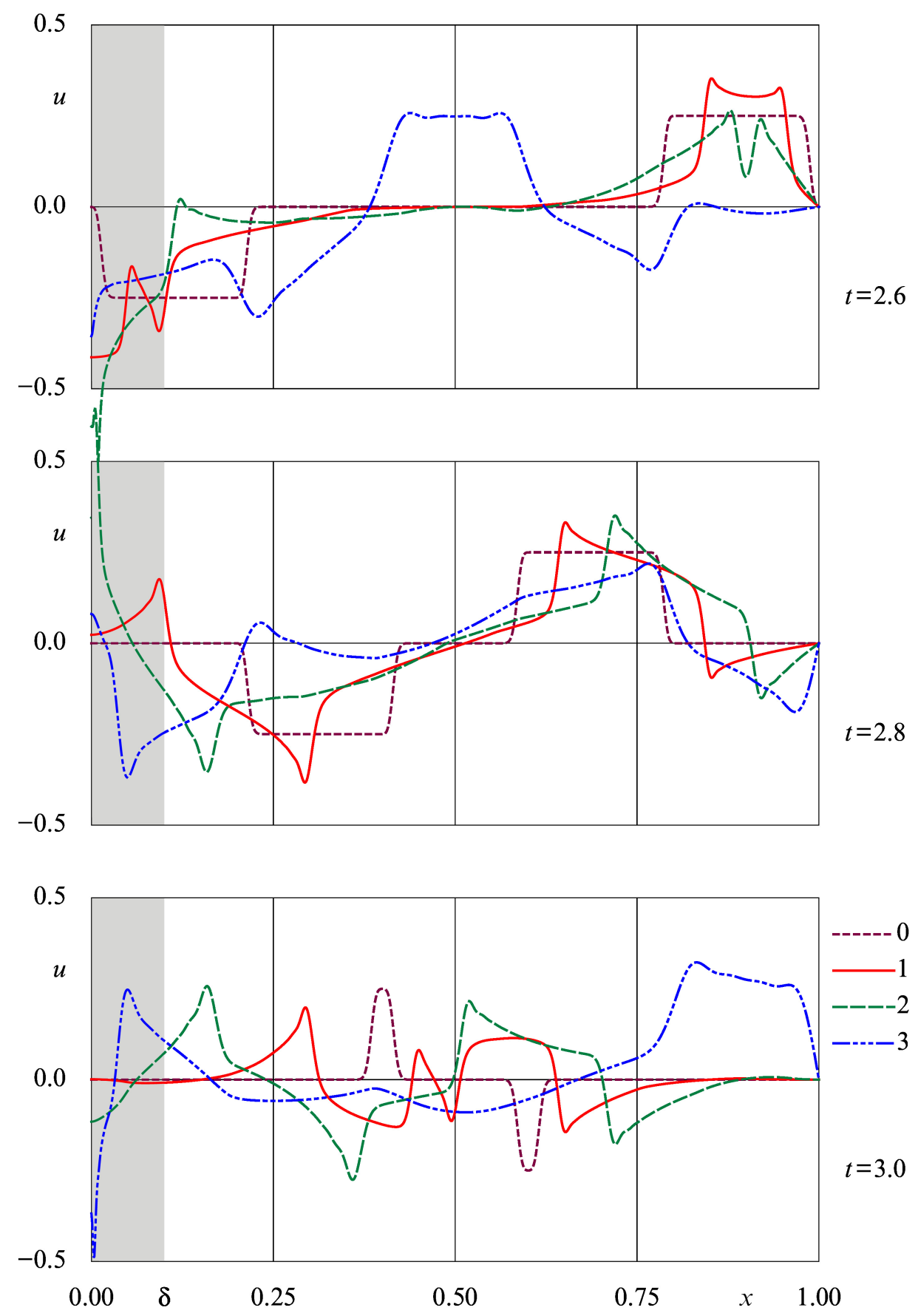

Fig. 8.9. Numerical solutions to four initial boundary value problems for wave equation (2.1) using approximation (5.6) (completion) 


\section{References}

1. S. R. Chakravarthy, D. A. Anderson, M. D. Salas, The split coefficient matrix method for hyperbolic systems of gas dynamic equations, AIAA Paper, 80-268 (1980).

2. S. R. Chakravarthy, Euler equations - implicit schemes and boundary conditions, AIAA J., 21(5) (1983), 699-706.

3. A. JefFrey, T. TANIUti, Non-linear wave propagation with applications to physics and magnetohydrodynamics , Academic Press, NY, London, 1964.

4. P. I. Kogut, O. P. Kupenko, G. Leugering, On existence of facilitated solutions to an optimal control problem for very strong degenerate wave equation, (in press).

5. V.P. Kolgan, Application of the principle of minimizing the derivative to the construction of finite-difference schemes for computing discontinuous solutions of gas dynamics, Scientific Notes of TsAGI, (3)(1972), 68-77.

6. B. VAN LEER, Towards the ultimate conservative difference scheme, $V$. A second order sequel to Godunov's method, J. Comput. Phys., 32(1) (1979), 101-136.

7. B. VAN LEER, Towards the ultimate conservative difference scheme, $V$. A second order sequel to Godunov's method, J. Comput. Phys., 135(2) (1997), 229-248.

8. B. VAN LEeR, A historical oversight: Vladimir P. Kolgan and his high-resolution scheme, J. Comput. Phys., 230(7), (2011), 2378-2383.

9. R. W. MACCORMACK, The effect of viscosity in hypervelocity impact cratering, AIAA Paper, 69-354 (1969).

10. R. W. MACCORMACK, The effect of viscosity in hypervelocity impact cratering, In: D. A. Caughey, M. M. Hafez (Eds.), Frontiers of Computational Fluid Dynamics 2002, World Scientific, New Jersey, London, 2001, 27-43.

11. J. L. Steger, R. WARMing, Flux vector splitting of the inviscid gas dynamic equations with application to finite-difference methods, J. Comput. Phys., 40(2), (1981), $263-293$.

12. G. B. Whitham, Linear and Nonlinear Waves, John Wiley and Sons, NY, 1974. 